AJOB Neuroscience

\title{
Deaf, Not Invisible: Sign Language Interpreting in a Global Pandemic
}

\section{Joanna Trzeciak Huss \& John Huss}

To cite this article: Joanna Trzeciak Huss \& John Huss (2021) Deaf, Not Invisible: Sign Language Interpreting in a Global Pandemic, AJOB Neuroscience, 12:4, 280-283, DOI: 10.1080/21507740.2021.1941410

To link to this article: https://doi.org/10.1080/21507740.2021.1941410

\section{Published online: 27 Oct 2021.}

Submit your article to this journal $\sqsubset$

Q View related articles $₫$

View Crossmark data $\asymp$ 


\title{
Deaf, Not Invisible: Sign Language Interpreting in a Global Pandemic
}

\author{
Joanna Trzeciak Huss ${ }^{a}$ (D) and John Huss ${ }^{b}$ (ID \\ ${ }^{\mathrm{a}}$ Kent State University; ${ }^{\mathrm{b}}$ University of Akron
}

One of us is in translation and interpreting studies, and has written on collaborative translation, and the other in philosophy of medicine. In our open peer commentary, we will focus attention on the two medical interpreters in Koutsouras, Eastwood, and Krishnamurthy (2021) as more than passive conduits for medical information between physician and patient, using a communication model from translation and interpreting studies that takes seriously the agency of the medical interpreter, considered not as a "hired hand," and not merely as part of the therapeutic alliance, but also as a frontline healthcare worker, a person deserving of ethical consideration. In the case study, sign language and cultural differences are described as impediments to communication -perhaps due to a tendency to pathologize the $\mathrm{D} / \mathrm{HH}(\mathrm{D} /$ deaf and hard of hearing) rather than to regard them as a linguistic minority -, whereas under an appropriate communication model, they comprise the basis for communication. In addition, we wish to draw attention to medical interpreters as especially vulnerable during the time of the covid-19 pandemic, where the interpreters may feel it necessary to remove their mask to assure full communication through facial expressions. Also, as participants in an end-of-life consultation, interpreters are subject to vicarious trauma through a process of neuroreciprocity. Overall, at a time of unprecedented visibility of sign language interpreters in public health, the invisibility of medical interpreters for the deaf in this case study presents a serious ethical blindspot. Here we raise some of these ethical issues, as well as bring to bear insights from the literature of translation and interpreting studies which we believe will open up fruitful new lines of communication between neurosurgeons, bioethicists, and neuroethicists and scholars, theorists, and practitioners of cross-cultural medical communication.

Despite the centrality of medical interpreters to nearly any cross-linguistic medical encounter and the increased prominence of signed language interpreting in public health communications during the Covid-19 pandemic, there is a dearth of discussion of medical interpreting in the bioethics literature. In Koutsouras, Eastwood, and Krishnamurthy (2021), the presence of two signed language interpreters in the described endof-life consultation is treated as a potential obstacle to successful communication, a source of anxiety over whether the physician's message is being clearly communicated to the deaf patient/surrogate.

\section{MODELS OF COMMUNICATION IN MEDICAL INTERPRETING}

The implicit communication model in Koutsouras, Eastwood, and Krishnamurthy (2021) is the conduit or transmission model, whose unidirectionality and lack of dynamic interaction have several consequences: instrumentalizing the medical interpreters; failing to recognize their corporeality, moral considerability, and moral agency; and ultimately undermining the effectiveness of the communication itself. If the primary goal of the end-of-life consultation is to ensure that consent is informed, and the secondary goal is to reassure the patient/surrogate, then a different communicative model is called for. In situations involving medical interpreting, the co-participant model reflects the actual praxis and ought to be explicitly identified and followed (Angelelli 2019). Signed language discourse is highly interactive, with different norms of interruption, turn-taking, requests for clarification, contextualization, and other dynamic interchanges that do not fit a model of transmitting a signal to a receiver. Indeed, in order for effective communication to occur, the interpreter needs to be a co-participant in the interpreted medical encounter, at the very least to provide clarification, to troubleshoot, and to serve as cross-cultural broker. Many medical interpreters, when asked, consider themselves part of the healthcare team, whose role is to help the provider deliver healthcare to patients (Angelelli 2019). Out of those 
present in the interpreted medical encounter, the interpreter will ordinarily be the one most familiar with the patient's/surrogate's culture and its norms, thus potentially able to aid in the goal of achieving informed consent and attempting to help the patient/ surrogate absorb the news without adding to the trauma.

\section{INVISIBLE AND DISEMBODIED INTERPRETERS}

To an increasing degree, interpreting is understood as an embodied practice, signed language interpreting all the more so. Consequently, it is striking in Koutsouras, Eastwood, and Krishnamurthy (2021) that we know the genders of the neurosurgery resident, the patient, his wife/surrogate, and eldest child, but we learn nothing of the gender, race, or ethnicity of the two medical interpreters. Gender, race, and ethnicity are among the variables at work in the power dynamics of medical interpreting, and hence are practically, sociologically, and analytically relevant (Swabey, Nicodemus, and Moreland 2014). From Koutsouras, Eastwood, and Krishnamurthy (2021), we know little about the physical parameters of the end-of-life consultation, and nothing about its duration. The two interpreters even lack monikers, for example, DI-1 and ASLI. We also know neither their age nor experience. We are not even told whether the interpreters present in the room were given the opportunity to physically distance, and whether they were wearing masks while signing, which is both medically and linguistically relevant.

\section{TEAM INTERPRETING}

According to Koutsouras, Eastwood, and Krishnamurthy (2021), the neurosurgery resident expresses anxiety that the presence of two interpreters could undermine effective relaying of the message. Yet research in interpreting studies suggests that, even in cases less linguistically complex than the one described, the presence of multiple interpreters may actually improve the quality, clarity, and accuracy of communication, especially when the consultation takes place over an extended period of time, or the interpreter in direct communication with the deaf person is a deaf interpreter (DI) or certified DI (CDI; Guardino 2018). To attain CDI certification from the Registry of Interpreters for the Deaf (RID; Registry of Interpreters for the Deaf, n.d.) is a rigorous undertaking. One must be deaf or hard-of-hearing, pass a practice test of interpreting skills, as well as a knowledge test to demonstrate understanding of deafness, the Deaf community, and Deaf culture. In addition to native or near-native fluency in ASL, certification designates training or expertise in the use of such communication-enhancing techniques as gesture, mime, drawing, and prop use (RID). Statistically, more than half of a DI's interpreting work involves collaboration with a hearing interpreter (Nicodemus and Taylor 2014, 91). While Koutsouras, Eastwood, and Krishnamurthy (2021) do not specify what level of certification the members of the interpreting team possessed, in end-of-life decisions where ethical nuance and emotional expressiveness are often essential to the encounter, the presence of a CDI is not only helpful but often necessary.

\section{PATHOLOGIZING THE DEAF}

In a clinical setting, there is a risk of pathologizing $\mathrm{D} /$ deaf users of signed languages or treating them as deficient rather than as a linguistic minority. In fact, the first language of most $\mathrm{D} /$ deaf individuals is signed language (in the United States, ASL and Black American Sign Language, or BASL), with English often a second language. Thus, ASL is not just a signed version of spoken English. They are linguistically distinct (morphologically, syntactically, etc.; Baker et al. 2016).

\section{THE INTERPRETER'S DILEMMA}

Medical interpreting for the deaf in the midst of the covid-19 pandemic raises specific challenges, as the corporeal requirements of sign language meet the medical reality of virus transmissibility. For example, one dilemma for the medical interpreter for the deaf is "to mask or not to mask." Facial expressions are of neurocognitive and linguistic significance not only for their emotive value, but also semantically and syntactically (Baker et al. 2016). In ASL and other signed languages, certain adjectives (e.g. big, small) and (especially) adverbs, have a non-manual form that involves different configurations of the lower facelips, mouth, cheeks (Baker et al. 2016). "Mouthings" are derivations from articulations of spoken words whereas "mouth gestures" are not (e.g. puffed cheeks to indicate "thick" in ASL; Baker et al. 2016). Thus, when ordinary surgical or N-95 masks obscure the lower face, they also obscure meaning, creating a communicative challenge for interpreters and patient alike. Consequently, interpreters may face the dilemma of compromising their ability to 
communicate by wearing a mask or exposing themselves to a health risk by removing their mask in the crowded environment of an end-of-life/palliative care consultation during Covid-19. To mitigate this problem, it may be advisable for the hospital to have available masks or $\mathrm{N}-95$ respirators that are transparent. These are in various stages of FDA approval and may partially mitigate the risk of virus transmission while allowing for better communication. And although interpreting via video remote technology is an option, from a sociolinguistic standpoint, it is a far less preferable option in the sensitive context of an end-of-life consultation. In addition, as a practical matter, video remote interpreting (VRI) requires a strong and reliable internet connection, not a given in the innards of a hospital, and moreover flattens a three-dimensional language, collapsing it into two dimensions (Sara Novic, personal communication).

\section{NEURO-RECIPROCITY AND VICARIOUS TRAUMA}

An occupational hazard for medical interpreters and others on the front lines of healthcare is vicarious trauma, which arises from exposure to the trauma of others (Guardino 2018). Investigations of vicarious trauma suggest it has a similar neurological basis to primary trauma (Isobel and Angus-Leppan 2018). Activity in the anterior insula, anterior cingulate cortex and inferior frontal cortex is the neurological correlate of an emotional experience. In vicarious trauma, it is thought that these same regions are activated via the mirror neuron system, resulting in neuro-reciprocity (Isobel and Angus-Leppan 2018). In the end-of-life consultation described by Koutsouras, Eastwood, and Krishnamurthy (2021), it can be presumed that Mrs. T, and possibly her son, are experiencing trauma. Hence, the medical interpreter, if empathically attuned to the state of Mrs. T., likely experienced a vicariously traumatic event, perhaps not the first or last in this line of work. As the parasympathetic nervous system attempts to regulate the response of an over-activated sympathetic nervous system, the perceived (mirrored) threat can elicit adaptive responses of the neuroendocrine, limbic, and autonomic systems (Isobel and Angus-Leppan 2018). Unless contained or resolved, such repeated responses, similar to those experienced by psychiatrists in their empathic engagement (Isobel and Angus-Leppan 2018), risk harming the medical interpreter (Guardino 2018). Harmful sequelae may be partially mitigated at the individual level by cultivating conscious awareness of these responses as they occur, engaging higher cortical centers in the contextualization of the trauma and at the institutional level through mutual support and trauma-informed approaches (Isobel and AngusLeppan 2018; Sansbury, Graves, and Scott 2015).

\section{MEDICAL ETHICS AND MEDICAL INTERPRETING}

As the Covid-19 pandemic makes evident, medical interpreters, and in this case sign language medical interpreters, are frontline healthcare workers and should be regarded as such. Rather than necessary channels, a condition for legal compliance, and a potential failed link in physician-patient communication, medical interpreters are part of an expanded team providing healthcare services to populations that are often quite vulnerable. Considering them as part of the therapeutic alliance draws attention not only to their skills but also to their needs. Medical interpreters may offer an expanded palate of situated cultural knowledge and communicative techniques, and are also moral agents and subjects of moral considerability in their own right. Especially in cases where interpreting or cross-language mediation is taking place, healthcare, medical communication and bioethics can benefit from advances in translation and interpreting studies.

\section{ACKNOWLEDGMENTS}

The authors would like to thank Brian James Baer, R. Kelly Washbourne, and Sara Novic for helpful feedback on the manuscript.

\section{ORCID}

Joanna Trzeciak Huss (D) http://orcid.org/0000-00018559-1556

John Huss (D) http://orcid.org/0000-0001-8372-0115

\section{REFERENCES}

Angelelli, C. V. 2019. Healthcare interpreting explained. New York, NY: Routledge.

Baker, A., van den Bogaerde, B., Pfau, R. and Schermer, T., eds. 2016. The linguistics of sign languages: An introduction. Amsterdam, the Netherlands: John Benjamins Publishing Company.

Guardino, D. L. 2018. Certified deaf interpreters' psychological well-being and coping mechanisms in medical situations. Order No. 11012625, Gallaudet University. https://www.proquest.com/dissertations-theses/certifieddeaf-interpreters-psychological-well/docview/2135720395/ se- 2 ? accountid $=9920$.

Isobel, S., and G. Angus-Leppan. 2018. Neuro-reciprocity and vicarious trauma in psychiatrists. Australasian Psychiatry: Bulletin of Royal Australian and New Zealand 
College of Psychiatrists 26 (4):388-90. doi:10.1177/ 1039856218772223

Koutsouras, G. W., G. Eastwood, and S. Krishnamurthy. 2021. An unusual conversation about dying during the COVID-19 pandemic: A neurosurgery resident's experience. $A J O B$ Neuroscience 12 (4): 278-79. doi:10.1080/ 21507740.2021 .1918283$.

Nicodemus, B., and M. Taylor. 2014. Deaf and hearing interpreting team preparation: A study using conversation analysis. In Deaf interpreters at work: International insights, ed. R. Adam, C. Stone, S. D. Collins, and M. Metzger, 90-116. Washington, DC: Gallaudet University Press.
Registry of Interpreters for the Deaf. n.d.. "Certified Deaf Interpreter (CDI)." https://rid.org/rid-certification-overview/available-certification/cdi-certification/

Sansbury, B. S., K. Graves, and W. Scott. 2015. Managing traumatic stress responses among clinicians: Individual and organizational tools for self-care. Trauma 17 (2): 114-22.

Swabey, L., B. Nicodemus, and C. Moreland. 2014. An examination of medical interview questions rendered in American Sign Language by deaf physicians and interpreters. In Investigations in healthcare interpreting, ed. B. Nicodemus and M. Metzger, 104-127. Washington, DC: Gallaudet University Press.

\title{
The Family as a Unit of Care in End-of-Life Conversations
}

\author{
Ben Sarbey
}

Duke University

End-of-life conversations with family members are frequently emotionally fraught and place physicians in situations they might not know how to handle. The conversation about dying from Koutsouras et al. (2021) demonstrates the need for neurologists and those in other specialized disciplines to receive enhanced training in family support. This stems from recognizing the family as a unit of care in end-of-life conversations, especially during public health emergencies. Neurologists must be prepared to provide basic grief management and emotional support to family members of their patients.

Neurologists are often a primary care provider for patients that are approaching the end of life, as well as a primary point of interaction for family members. A consensus review by the European Academy of Neurology and the European Association for Palliative Care found that support of family caregivers is indispensable to "reduce complicated bereavement and improve patients' quality of life" (Oliver et al. 2016). The American Academy of Neurology insisted as early as 1996 that "it is imperative that neurologists understand, and learn to apply, the principles of palliative medicine" ("Palliative Care in Neurology. The American Academy of Neurology Ethics and
Humanities Subcommittee" 1996). But there is still a significant gap in the training and provision of pain and symptom management as well as adequate family care by neurologists (Boersma et al. 2014). Neurologists and those in other disciplines need to incorporate the skills and approaches of palliative care into their own caregiving.

Palliative care is not just for specialists in palliative medicine. Basic pain management, discussion of endof-life prognoses, and family support are skills that physicians in all specialties that work with patients should have. So-called "generalist" palliative care is increasingly recognized as a vital element of the training and practice of physicians in neurology (Borasio 2013; Quill and Abernethy 2013). Patients in neurology have unique palliative needs stemming from the relative lack of cures for neurologic disorders, the long, gradual decline typical of these disorders, as well as unique symptom profiles and psychosocial issues involved (Boersma et al. 2014). Neurologists must hold themselves accountable for providing effective end-of-life treatment to patients with palliative needs as well as their families.

Koutsouras et al. highlight the need for the kinds of skills involved in generalist palliative care in the 